\title{
Impact of Transceiver Hardware Impairments on the Ergodic Channel Capacity for Rayleigh-Product MIMO Channels
}

\author{
Anastasios Papazafeiropoulos*, Shree K. Sharma ${ }^{\dagger}$, Symeon Chatzinotas ${ }^{\dagger}$, Thar Ratnarajah*, and Björn Ottersten ${ }^{\dagger}$ \\ *Institute for Digital Communications, University of Edinburgh, Edinburgh EH9 3JL, U.K. \\ ${ }^{\dagger}$ SnT - securityandtrust.lu, University of Luxembourg, Luxembourg \\ Email: \{a.papazafeiropoulos, t.ratnarajah@ed.ac.uk\}@ed.ac.uk, \{shree.sharma, \\ symeon.chatzinotas,bjorn.ottersten\}@uni.lu
}

\begin{abstract}
This paper aims at a realistic evaluation of Rayleighproduct multiple-input multiple-output (MIMO) systems. Specifically, by considering the residual transceiver hardware impairments into account, we derive the ergodic channel capacity of a MIMO system with optimal receivers in the case of insufficient scattering. Actually, motivated by the increasing interest for massive MIMO systems, we investigate the impact of transceiver hardware imperfections in systems with both finite (conventional) and large number of antennas under rank deficient channel matrix conditions by varying the severity of hardware quality. Among the interesting outcomes, we emphasize that the residual hardware transceiver impairments result to a saturation of the ergodic channel capacity within the high signal-to-noise ratio (SNR) regime. Furthermore, we observe that the higher the "richness" of the scattering environment, the higher the ergodic channel capacity till it gets saturated.
\end{abstract}

\section{INTRODUCTION}

Remarkable benefits coming from the employment of multiple-input multiple-output (MIMO) systems such as increased spectral efficiency has attracted great scientific interest in academia [1]. Moreover, its establishment has been grounded by means of industrial applications and wireless communication standards including IEEE 802.11n (Wi-Fi), IEEE 802.11ac (Wi-Fi), HSPA+ (3G), WiMAX, and Long Term Evolution systems.

Altough most works assume "rich" scattering conditions, described by a full rank matrix, certain environments result to a rank deficient matrix due to insufficient scattering [2]. This phenomenon is known as double scattering effect [2], [3], and it has been experimentally validated in [4]. Especially, when the antenna elements and the scatterers are sufficiently separated, the mathematical description is given by the Rayleighproduct model [5]-[7].

In this context, the corresponding literature is based on the strong unrealistic assumption of ideal transceiver hardware. Towards a practical investigation of wireless communication systems, the residual additive transceiver hardware impairments are going to be addressed in this work. These impairments denote the aggregate effect from many impairments and are modelled as independent and additive distortion Gaussian noise at both sides of the system. Basically, they arise after the application of inadequate compensation algorithms due to imperfect parameters estimation caused by the randomness and the time variation of the hardware characteristics and by inaccurate models because of unsophisticated compensation algorithms with limited precision [8], [9].

Recently, several studies have investigated the effects of additive transceiver hardware impairments in several types of channels and systems such as Rayleigh, Rician fading channels and relay systems [8]-[12]. In this direction, we focus not only on conventional MIMO systems, but also on the promising massive MIMO technology for the fifth generation (5G) systems [13], [14]. Actually, it becomes more crucial to investigate the impact of additive transceiver hardware impairments in the large number of antennas regime, since massive MIMO is attractive if its implementation takes place with low-cost hardware, which is more prone to hardware impairments [15]-[17].

To the best of our knowledge, there appears no investigation of the effects of residual transceiver impairments in the case of Rayleigh-product channels. Hence, we extend the work of [15] dealing with Rayleigh MIMO systems to Rayleigh-product MIMO channels by introducing the residual hardware impairments. Moreover, we analyze the impact of these impairments on both finite and large MIMO systems, where the numbers of antennas and scatterers tend to infinity, but with a given ratio. Especially, we derive a closed-form expression for the ergodic capacity, when the number of antennas is finite, and we verify the theoretical results by using simulations. Furthermore, we investigate how the number of scatterers interact with the quality of hardware.

The remainder of this paper is organized as follows: Section II presents the system and signal models for Rayleighproduct channels in the case of both ideal and impaired hardware cases. In Section III, we provide a study of the sumrates for the aforementioned channels with optimal receivers, when the number of antennas is finite. Section IV presents the investigation of Rayleigh-product MIMO channels in the presence of hardware impairments in the large system limit. In Section V, numerical results illustrate the impact residual hardware impairments in finite and asymptotically large numbers of antennas regimes. Finally, concluding remarks are given in Section VI.

Notation: Vectors and matrices are denoted by boldface lower and upper case symbols. The transpose, Hermitian transpose, and trace operators are represented by $(\cdot)^{\mathrm{T}},(\cdot)^{\mathrm{H}}$, 
and $\operatorname{tr}(\cdot)$, respectively. The expectation operator and the determinant of a matrix are denoted by $\mathbb{E}[\cdot]$ and $\operatorname{det}(\cdot)$, respectively. The notations $\mathcal{C}^{M}$ and $\mathcal{C}^{M \times N}$ refer to complex $M$-dimensional vectors and $M \times N$ matrices, respectively. The $\operatorname{diag}\{\cdot\}$ operator generates a diagonal matrix from a given vector. Finally, $\mathbf{b} \sim \mathcal{C N}(\mathbf{0}, \boldsymbol{\Sigma})$ denotes a circularly symmetric complex Gaussian with zero-mean and covariance matrix $\Sigma$, while $(\cdot)^{+}$signifies the positive part of its argument.

\section{System ModeL}

Consider a flat-fading point-to-point MIMO channel with $M$ and $N$ transmit and receive antennas, respectively. The received signal is described by

$$
\mathbf{y}=\mathbf{H x}+\mathbf{z},
$$

where $\mathbf{x} \in \mathcal{C}^{M \times 1}$ denotes the zero-mean transmit Gaussian vector with covariance matrix $\mathbb{E}\left[\mathbf{x x}^{\mathrm{H}}\right]=\mathbf{Q}=\frac{\rho}{M} \mathbf{I}_{M}$, and $\mathbf{z} \sim \mathcal{C N}\left(\mathbf{0}, \mathbf{I}_{N}\right)$ is the AWGN noise vector at the receiver. Focusing on Rayleigh-product MIMO channels, $\mathbf{H} \in \mathbb{C}^{N \times M} \sim$ $\mathcal{C N}\left(\mathbf{0}, \mathbf{I}_{N} \otimes \mathbf{I}_{M}\right)$ is described as

$$
\mathbf{H}=\frac{1}{\sqrt{K}} \mathbf{H}_{1} \mathbf{H}_{2},
$$

where $\mathbf{H}_{1} \in \mathbb{C}^{N \times K} \sim \mathcal{C N}\left(\mathbf{0}, \mathbf{I}_{N} \otimes \mathbf{I}_{K}\right)$ and $\mathbf{H}_{2} \in \mathbb{C}^{K \times M} \sim$ $\mathcal{C N}\left(\mathbf{0}, \mathbf{I}_{K} \otimes \mathbf{I}_{M}\right)$ are random matrices with $K$ quantifying the number of scatterers in the propagation environment [5]. The physical representation of this channel model includes flatfading under the presence of a certain number of independent scatterers.

Despite that hardware impairments are inevitable, their impact on Rayleigh-product MIMO channels has not been studied in the literature. This unrealistic assumption leads to misleading calculations with deceiving results. Actually, both the transmitter and the receiver are affected by certain inevitable additive impairments such as I/Q imbalance and high power amplifier (HPA) non-linearities [8]. Although mitigation schemes at both the transmitter and the receiver are included during the design and implementation, residual impairments, modelled as additive distortion noises, are unavoidable [8], [9]. As a direct effect, hardware transmit impairments induce a mismatch between the intended signal and what is actually transmitted during the transmit processing. Similarly, a distortion of the received signal at the receiver side is produced due to imperfect receiver hardware.

Incorporating the residual additive transceiver impairments to (1), the mathematical characterization of a more practical channel model is given by

$$
\mathbf{y}=\mathbf{H}\left(\mathbf{x}+\boldsymbol{\eta}_{\mathrm{t}}\right)+\boldsymbol{\eta}_{\mathrm{r}}+\mathbf{z},
$$

where the additive terms $\boldsymbol{\eta}_{\mathrm{t}}$ and $\boldsymbol{\eta}_{\mathrm{r}}$ denote the distortion noises coming from the residual impairments in the $M$ antennas transmitter and $N$ antennas receiver, respectively. Based on a general approach, validated by measurement results, the transmitter and receiver distortion noises are Gaussian distributed with their average power being proportional to the average signal power [9]. More concretely, the distortion noises are modelled as

$$
\begin{aligned}
\boldsymbol{\eta}_{\mathrm{t}} & \sim \mathcal{C N}\left(\mathbf{0}, \delta_{\mathrm{t}}^{2} \operatorname{diag}\left(q_{1}, \ldots, q_{M}\right)\right) \\
\boldsymbol{\eta}_{\mathrm{r}} & \sim \mathcal{C N}\left(\mathbf{0}, \delta_{\mathrm{r}}^{2} \operatorname{tr}(\mathbf{Q}) \mathbf{I}_{N}\right),
\end{aligned}
$$

where $\delta_{\mathrm{t}}^{2}$ and $\delta_{\mathrm{r}}^{2}$ are proportionality parameters characterizing the severity of the residual impairments at the transmitter and the receiver. Given that the covariance matrix $\mathrm{Q}$ equals to $\frac{\rho}{M} \mathbf{I}_{M}$, the additive transceiver impairments are written as

$$
\begin{aligned}
\boldsymbol{\eta}_{\mathrm{t}} & \sim \mathcal{C N}\left(\mathbf{0}, \delta_{\mathrm{t}}^{2} \frac{\rho}{M} \mathbf{I}_{M}\right), \\
\boldsymbol{\eta}_{\mathrm{r}} & \sim \mathcal{C N}\left(\mathbf{0}, \delta_{\mathrm{r}}^{2} \rho \mathbf{I}_{N}\right) .
\end{aligned}
$$

In practical applications such as long term evolution (LTE), the values of $\delta_{\mathrm{t}}, \delta_{\mathrm{r}}$ range in the interval $[0.08,0.175][18, \mathrm{Sec}$. 14.3.4].

\section{FINITE ANALYSIS}

This section presents the ergodic channel capacity of Rayleigh-product MIMO channels with optimal linear receivers by including the residual transceiver hardware impairments, when the number of antennas is finite. Specifically, in the case of Gaussian additive impairments, the following proposition allows us to express the ergodic capacity, when optimal receivers are employed.

Proposition 1: The ergodic channel capacity of a practical Rayleigh-product MIMO channel with optimal linear receivers, but with residual transceiver impairments under the constraint $\operatorname{tr}(\mathbf{Q}) \leq \rho$ is described by

$$
\mathrm{C}^{\text {opt }}\left(\rho, M, N, K, \delta_{\mathrm{t}}, \delta_{\mathrm{r}}\right)=\mathbb{E}\left[\log _{2} \operatorname{det}\left(\mathbf{I}_{N}+\frac{\rho}{M} \mathbf{H H}^{\mathrm{H}} \boldsymbol{\Phi}^{-1}\right)\right],
$$

where $\boldsymbol{\Phi}=\frac{\rho}{K M} \delta_{\mathrm{t}}^{2} \mathbf{H}_{1} \mathbf{H}_{2} \mathbf{H}_{2}^{\mathrm{H}} \mathbf{H}_{1}^{\mathrm{H}}+\left(\rho \delta_{\mathrm{r}}^{2}+1\right) \mathbf{I}_{N}$.

Proof: It can be observed that (3) is an instance of the standard MIMO channel given by (2) for any channel realizations $\mathbf{H}_{1}, \mathbf{H}_{2}$ and transmit signal covariance matrix $\mathbf{Q}$, being a scaled identity matrix, but with a different noise covariance given by

$$
\begin{aligned}
\boldsymbol{\Phi} & =\frac{\delta_{\mathrm{t}}^{2}}{K} \mathbf{H}_{1} \mathbf{H}_{2} \operatorname{diag}\left(q_{1}, \ldots, q_{M}\right) \mathbf{H}_{2}^{\mathrm{H}} \mathbf{H}_{1}^{\mathrm{H}} \\
& +\left(\delta_{\mathrm{r}}^{2} \operatorname{tr} \mathbf{Q}+1\right) \mathbf{I}_{N} .
\end{aligned}
$$

Subsequently, the ergodic capacity is written as

$$
\mathrm{C}^{\text {opt }}(\rho, M, N, K)=\max _{\mathbf{Q}: \operatorname{tr} \mathbf{Q} \leq \rho} \mathbb{E}\left[\log _{2} \operatorname{det}\left(\mathbf{I}_{N}+\mathbf{H} \mathbf{Q} \mathbf{H}^{\mathrm{H}} \boldsymbol{\Phi}^{-1}\right)\right] .
$$

Taking into account for the sufficiency and optimality of the input signal $\mathbf{x}$, since it is Gaussian distributed with covariance matrix $\mathbf{Q}=\frac{\rho}{M} \mathbf{I}_{M}$ [1], the proof is concluded.

In what follows, we refer to $p=\max (M, N), q=$ $\min (M, N), t=\max (q, K), s=\min (K, M, N), m=$ median $(K, M, N)$, and $\tilde{\delta}_{\mathrm{t}}^{2}=1+\delta_{\mathrm{t}}^{2}$ for notational convenience.

The following theorem presents the ergodic capacity of Rayleigh-product channels with optimal receivers in the presence of additive Gaussian hardware impairments in closed form, which is one of the main contributions of this paper.

Theorem 1: The ergodic capacity of practical Rayleighproduct MIMO channels with optimal receivers in the finite number of antennas regime, accounting for residual additive Gaussian hardware transceiver impairments, is given by

$\mathrm{C}^{\mathrm{opt}}(\rho, M, N, K)=\mathcal{A}\left(\mathcal{C}_{1}(\rho, M, N, K)-\mathcal{C}_{2}(\rho, M, N, K)\right)$, 
where

$$
\mathcal{A}=\frac{\mathcal{K}}{\ln 2} \sum_{i=1}^{s} \sum_{j=1}^{s} \frac{G_{i, j}}{\Gamma(p-s+j)}
$$

with

$$
\mathcal{K}=\left(\prod_{i=1}^{s} \Gamma(s-i+1) \Gamma(t-i+1)\right)^{-1},
$$

and $G_{i, j}$ is the $(i, j)$ th cofactor of a $s \times s$ matrix $\mathbf{G}$ whose $(u, v)$ th entry is

$$
[G]_{u, v}=\Gamma(t-s+u+v-1) .
$$

The terms, represented by $\mathrm{C}_{i}$ for $i=1,2$, are given by

$$
\mathcal{C}_{i}(\rho, M, N, K)=G_{4,2}^{1,4}\left(z_{i} \mid \begin{array}{c}
a_{1}, a_{2}, 1,1 \\
1,0
\end{array}\right),
$$

where $z_{1}=\frac{\frac{\rho}{K N} \tilde{\delta}_{t}^{2}}{\rho \delta_{\mathrm{r}}^{2}+1}, z_{2}=\frac{\frac{\rho}{K N} \delta_{t}^{2}}{\rho \delta_{\mathrm{r}}^{2}+1}, a_{1}=s+2-i-j-t$, and $a_{2}=s+1-p-j$. Note that $\Gamma(z)=\int_{0}^{\infty} t^{z-1} e^{-t} \mathrm{~d} t$ and $G_{p, q}^{m, n}\left(x \mid \begin{array}{c}\alpha_{1}, \ldots, \alpha_{p} \\ \beta_{1} \ldots, \beta_{q}\end{array}\right)$ are the Gamma function [19, Eq. 8.310] and the Meijer's G-function [19, Eq. 9.301], respectively.

Proof: See Appendix B.

\section{Asymptotic Analysis}

In this section, we investigate the effects of transceiver hardware impairments on the ergodic channel capacity of Rayleighproduct MIMO channels when the system dimensions tend asymptotically to infinity. Having in mind that free probability (FP) analysis and deterministic equivalent analysis require a polynomial solution and solutions of fixed-point equations, herein, we employ the FP method, in order to shed light on the large number of antennas regime. Although the results are tight approximations, becoming exact as the system dimensions become large, numerical results presented later in Section V and the existing literature show that they are valid even for a finite system size [11], [12], [20].

In the large system limit, we can obtain the corresponding asymptotic limit of the capacity per receive antenna while keeping the system dimensions in terms of their ratios fixed, i.e., $\beta$ and $\gamma$ are kept fixed. Hence, we write (8) as $\tilde{\mathrm{C}}^{\mathrm{opt}}\left(\rho, \beta, \gamma, \delta_{\mathrm{t}}, \delta_{\mathrm{r}}\right)=\tilde{\mathrm{C}}_{1}^{\mathrm{opt}}\left(\rho, \beta, \gamma, \delta_{\mathrm{t}}, \delta_{\mathrm{r}}\right)-\tilde{\mathrm{C}}_{2}^{\mathrm{opt}}\left(\rho, \beta, \gamma, \delta_{\mathrm{t}}, \delta_{\mathrm{r}}\right),($

where $\tilde{\mathrm{C}}_{i}^{\text {opt }}$ for $i=1,2$ is given by

$$
\begin{aligned}
\tilde{\mathrm{C}}_{i}^{\text {opt }} & =\frac{1}{N} \lim _{K, M, N \rightarrow \infty} \mathbb{E}\left[\log _{2} \operatorname{det}\left(\mathbf{I}_{K}+f_{i} \mathbf{H}_{2} \mathbf{H}_{2}^{\mathrm{H}} \mathbf{H}_{1}^{\mathrm{H}} \mathbf{H}_{1}\right)\right. \\
& =\frac{K}{N} \lim _{K, M, N \rightarrow \infty} \mathbb{E}\left[\frac{1}{K} \sum_{j=1}^{K} \log _{2}\left(1+f_{i} K \lambda_{j}\left(\frac{1}{K} \mathbf{K}\right)\right)\right] \\
& \rightarrow \gamma \int_{0}^{\infty} \log _{2}\left(1+f_{i} K x\right) f_{\frac{\mathrm{K}}{K}}^{\infty}(x) \mathrm{d} x,
\end{aligned}
$$

where $\lambda_{j}\left(\frac{1}{K} \mathbf{K}\right)$ is the $j$ th ordered eigenvalue of matrix $\frac{1}{K} \mathbf{K}$, and $f_{\frac{1}{K}}^{\infty} \mathbf{K}$ denotes the asymptotic eigenvalue probability density function (a.e.p.d.f.) of $\frac{1}{K} \mathbf{K}$.

The following variable definitions allow us to simplify the analysis. Specifically, we denote

$$
\begin{aligned}
\tilde{\mathbf{N}}_{1} & =\mathbf{H}_{1}^{\mathrm{H}} \mathbf{H}_{1} \\
\tilde{\mathbf{N}}_{2} & =\mathbf{H}_{2} \mathbf{H}_{2}^{\mathrm{H}} \\
\mathbf{K} & =\tilde{\mathbf{N}}_{2} \tilde{\mathbf{N}}_{1} .
\end{aligned}
$$

Theorem 2: The ergodic capacity of Rayleigh-product MIMO channels for optimal receivers in the presence of additive Gaussian transceiver impairments, when the number of transmit and receive antennas $(M$ and $N)$ as well as the number of scatterers $K$ tend to infinity with given ratios $\beta=\frac{M}{K}$ and $\gamma=\frac{K}{N}$, is given by

$$
\mathrm{C}^{\text {opt }}\left(\rho, \beta, \gamma, \delta_{\mathrm{t}}, \delta_{\mathrm{r}}\right) \rightarrow \gamma \int_{0}^{\infty} \log _{2}\left(\frac{1+f_{1} K x}{1+f_{2} K x}\right) f_{\frac{\mathrm{K}}{K}}^{\infty}(x) \mathrm{d} x,
$$

where the a.e.p.d.f. of $\frac{K}{K} f_{\frac{K}{K}}^{\infty}$ is obtained by determining the imaginary part of its Stieltjes transform $\mathcal{S}$ for real arguments. The derivation of $\frac{\mathrm{K}}{K} f_{\frac{\mathrm{K}}{K}}^{\infty}$ is provided in Appendix C.

\section{Numerical Results}

In this section, we verify the theoretical analysis carried out in previous sections and illustrate the impact of impairments on the ergodic capacity of Rayleigh-product MIMO channels with optimal linear receivers.

\section{A. Finite Analysis}

Herein, we present numerical results corresponding to the analysis presented in Section III.

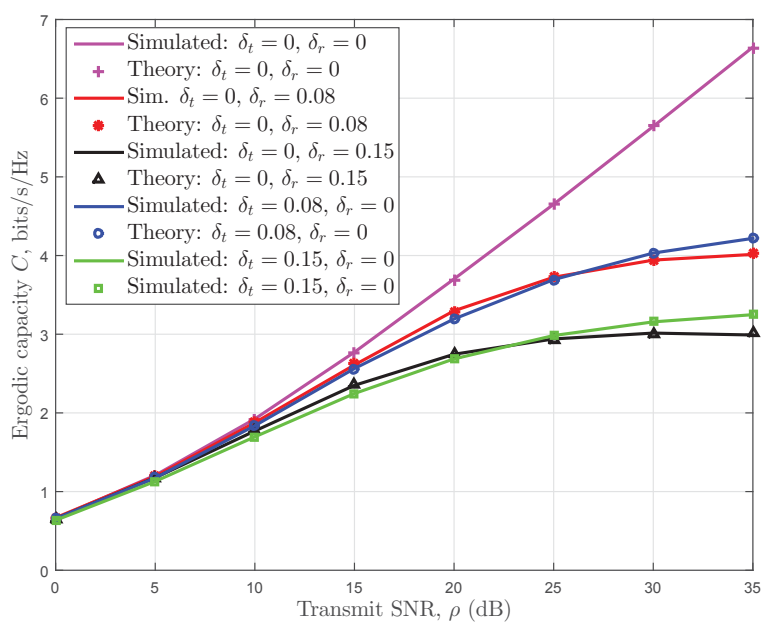

Fig. 1. Per-antenna ergodic capacity of Rayleigh-product MIMO channels with optimal receivers for different levels of impairment severity at the transmitter and receiver $(K=3, M=4, N=5)$.

Fig. 1 presents the per-antenna ergodic capacity of Rayleighproduct channels with optimal receivers considering $K=3$, $M=4, N=5$. Both theoretical and simulated results are presented for the cases with and without residual transceiver hardware impairments. The theoretical curve for the case without impairments was obtained by following the analysis considered in [7]. Whereas, the theoretical curves for the practical case with hardware impairments were obtained by means of Theorem 1. Furthermore, the simulated curves were obtained by averaging the corresponding capacities over $10^{3}$ random instances of $\mathbf{H}_{1}$ and $\mathbf{H}_{2}$.

It can be noted from Fig. 1 that the proposed capacity expression matches well with the Monte Carlo (MC) simulation for arbitrary finite values of $K, M$, and $N$. Most importantly, we note that in the absence of residual hardware impairments, i.e., $\delta_{t}=0, \delta_{r}=0$, the per-antenna ergodic capacity increases 
monotonically with the increase in the value of $\rho$. On the contrary, in the presence of residual hardware impairments, the ergodic capacity first increases with the increase in the value of $\rho$ and then gets saturated after a certain value of $\rho$. Besides, the capacity gap in the presence of impairments as compared to the case without impairments (ideal hardware) increases with the increase in the value of $\rho$. Furthermore, another important observation from Fig. 1 is that the perantenna ergodic capacity decreases with the increase in the severity of the residual hardware impairments. In particular, the lower the quality of transceiver hardware (higher severity), the earlier the saturation point appears.

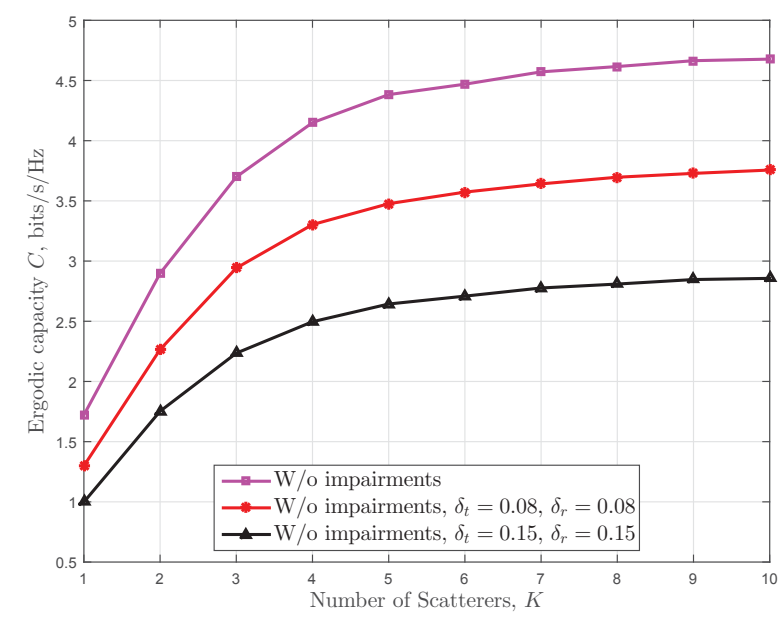

Fig. 2. Per-antenna ergodic capacity of Rayleigh-product MIMO channels versus the number of scatterers with optimal linear receivers $(\rho=20 \mathrm{~dB}$, $M=4, N=5$ ).

Furthermore, Fig. 1 also demonstrates the impact of different levels of impairments at the transmitter and receiver sides. In order to evaluate the effect of impairments present in one side (transmit/receive), the impairment value on the other side (receive/transmit) is set to be zero. It can be observed that at higher signal-to-noise ratio (SNR) values, the effect of $\delta_{r}$ is more severe than that of $\delta_{t}$, and this severity increases as the value of the corresponding impairment increases.

In order to illustrate the effect of the number of scatterers, we plot the per-antenna ergodic capacity versus $K$ in Fig. 2 considering $\rho=20 \mathrm{~dB}, M=4, N=5$. It can be noted that the per-antenna ergodic capacity first increases with the value of $K$ and then tends to saturate after its certain value. Furthermore, the per-antenna capacity versus $K$ decreases with the increase in the severity of the impairments. In other words, as the richness of scattering increases, the capacity for a certain quality of hardware increases and finally saturates. Also, the saturation with the variation in $K$ occurs earlier for the higher value of impairments.

\section{B. Asymptotic Analysis}

In order to validate our asymptotic analysis of the ergodic capacity of Rayleigh-product MIMO channels with optimal linear receivers presented in Section IV, we plot the a.e.p.d.f. of $\mathbf{K}$ in Fig.3, where the histogram represents the p.d.f. of the matrix $\mathbf{K}$ calculated numerically based on MC simulation. Furthermore, the solid line depicts the a.e.p.d.f. obtained by

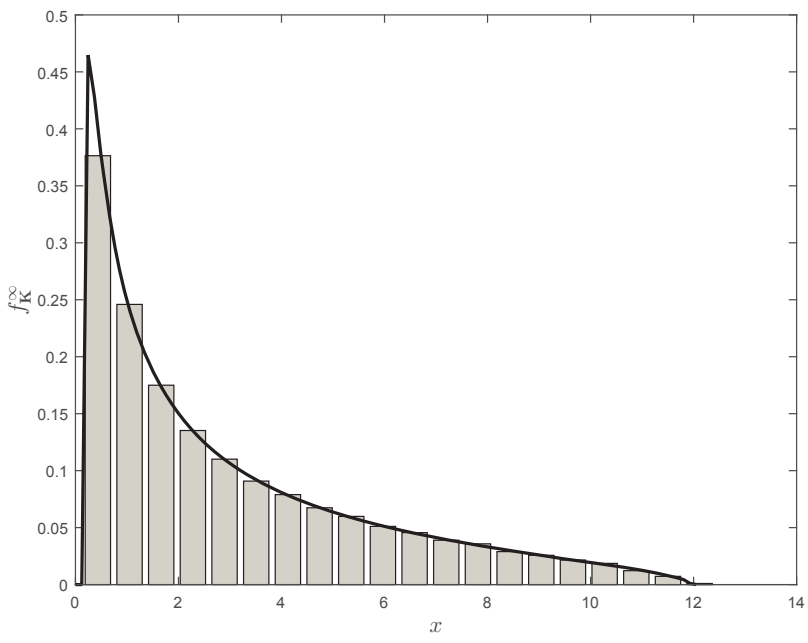

Fig. 3. A.e.p.d.f. of $\mathbf{K}$ in the case of Rayleigh-product MIMO channels $\left(\rho=20 \mathrm{~dB}, K=100, M=300, N=200, \delta_{t}=\delta_{r}=0.15\right)$.

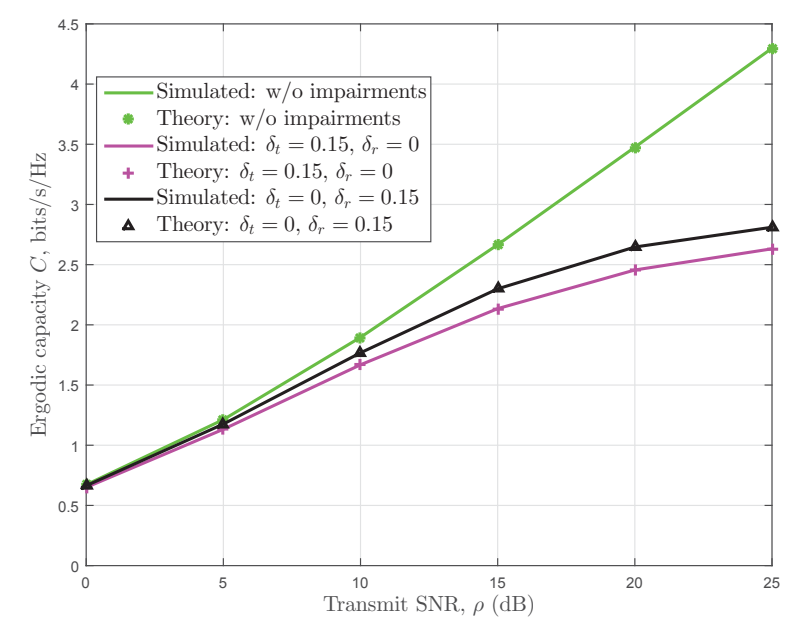

Fig. 4. Per-antenna ergodic capacity of Rayleigh-product MIMO channels versus $\rho$ in the large system regime $(K=100, M=300, N=200)$.

solving the polymonial (35) regarding the Stieltjes transform of the corresponding a.e.p.d.f., and then applying Lemma 3. A perfect agreement between the results obtained from theoretical analysis and MC simulation has been obtained as reflected in Fig. 3.

In Fig. 4, we plot the theoretical and simulated per-antenna ergodic capacities versus $\rho$ considering $K=100, M=300$, and $N=200$. Both the cases with and without impairments are presented. From the figure, it can be observed that theoretical and simulated capacity curves for both the considered cases match perfectly. Furthermore, as expected, the per-antenna capacity increases with the increase in the value of $\rho$ in the absence of impairments, i.e., $\delta_{t}=\delta_{r}=0$. However, as in the finite case, the per-antenna capacity saturates after a certain value of $\rho$ in the presence of impairments.

Fig. 5 depicts the per-antenna capacity of Rayleigh-product MIMO channels versus $\beta$ and $\gamma$ by considering $K=10, \rho=$ $20 \mathrm{~dB}, \delta_{t}=0.15, \delta_{r}=0.15$. It can be noted that the perantenna capacity increases with the increase in the values of $\beta$ and $\gamma$. Another important observation is that the rate of 


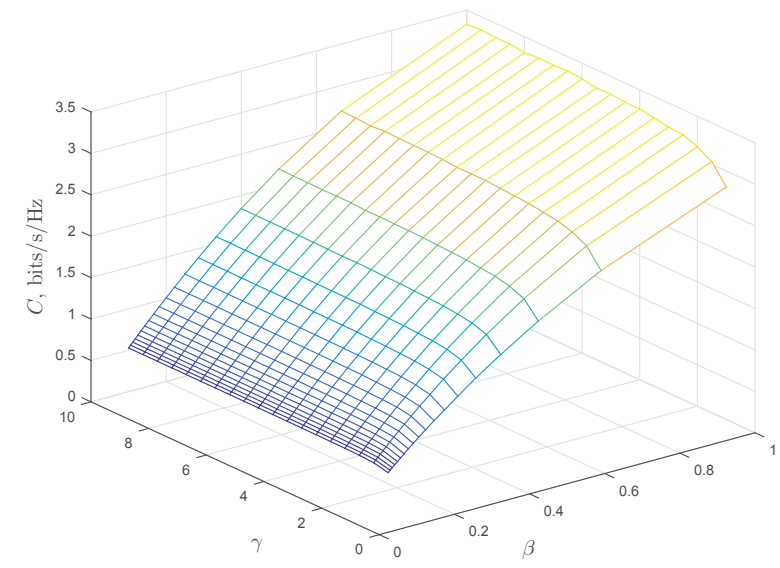

Fig. 5. Per-antenna ergodic capacity of Rayleigh-product MIMO channels versus $\beta$ and $\gamma$ in the large system regime $\left(K=10, \rho=20 \mathrm{~dB}, \delta_{t}=0.15\right.$, $\left.\delta_{r}=0.15\right)$.

capacity increase with respect to $\beta$ is much steeper than the capacity variation with $\gamma$.

\section{CONCLUSIONS}

This paper delved into the impact of residual transceiver hardware impairments on the performance of double Rayleigh MIMO channels. Analytical results regarding the ergodic capacity in the presence of residual transceiver impairments with optimal linear receivers were obtained. In particular, we investigated both conventional and large number of antennas regimes. Moreover, we characterized how the richness of the propagation environment affects the system performance, when the inevitable residual hardware impairments are taken into consideration. Most importantly, we shed light on the impact of hardware quality in rank-deficient channel conditions.

\section{APPENDIX A}

USEFUL LEMMAS

Herein, given the eigenvalue probability distribution function $f_{\mathbf{X}}(x)$ of a matrix $\mathbf{X}$, we provide useful definitions and lemmas that are considered during our analysis. In the following definitions, $\delta$ is a nonnegative real number.

Definition 1 ( $\eta$-transform [21, Definition 2.11]): The $\eta$ transform of a positive semidefinite matrix $\mathbf{X}$ is defined as

$$
\eta_{\mathbf{X}}(\delta)=\int_{0}^{\infty} \frac{1}{1+\delta x} f_{\mathbf{X}}(x) \mathrm{d} x .
$$

Definition 2: [S-transform [21, Definition 2.15]] The Stransform of a positive semidefinite matrix $\mathbf{X}$ is defined as

$$
\Sigma_{\mathbf{X}}(x)=-\frac{x+1}{x} \eta_{\mathbf{X}}^{-1}(x+1) .
$$

Definition 3 (The Marčenko-Pastur law density function [22]): Given a Gaussian $K \times M$ channel matrix $\mathbf{H} \sim$ $\mathcal{C N}\left(\mathbf{0}, \mathbf{I}_{K}\right)$, the a.e.p.d.f. of $\frac{1}{K} \mathbf{H} \mathbf{H}^{\mathrm{H}}$ converges almost surely (a.s.) to the non-random limiting eigenvalue distribution of the Marčenko-Pastur law given by

$$
f_{\frac{1}{K} \mathbf{H H}^{\mathrm{H}}}^{\infty}(x)=(1-\beta)^{+}(x)+\frac{\sqrt{(x-a)^{+}(b-x)^{+}}}{2 \pi x},
$$

where $a=(1-\sqrt{\beta})^{2}, b=(1+\sqrt{\beta})^{2}, \beta=\frac{M}{K}$, and $\delta(x)$ is Dirac's delta function.

Lemma 1 ( [21, Eqs. 2.87, 2.88]): The S-transform of the matrix $\frac{1}{K} \mathbf{H}^{\mathrm{H}} \mathbf{H}$ is expressed as

$$
\Sigma_{\frac{1}{K} \mathbf{H}^{\mathrm{H}} \mathbf{H}}(x, \beta)=\frac{1}{1+\beta x},
$$

while the S-transform of the matrix $\frac{1}{K} \mathbf{H H}^{\mathrm{H}}$ is obtained as

$$
\Sigma_{\frac{1}{K} \mathbf{H H}^{\mathrm{H}}}(x, \beta)=\frac{1}{\beta+x} .
$$

Lemma 2 ( [21, Eq. 2.48]): The Stieltjes-transform of a positive semidefinite matrix $\mathbf{X}$ can be derived by its $\eta$ transform according to

$$
\mathcal{S}_{\mathbf{X}}(x)=-\frac{\eta_{\mathbf{X}}(-1 / x)}{x} .
$$

Lemma 3 ( [21, Eq. 2.45]): The a.e.p.d.f. of $\mathbf{X}$ is obtained by the imaginary part of the Stieltjes transform $\mathcal{S}$ for real arguments as

$$
f_{\mathbf{X}}^{\infty}(x)=\lim _{y \rightarrow 0^{+}} \frac{1}{\pi} \mathfrak{I}\left\{\mathcal{S}_{\mathbf{X}}(x+\mathrm{j} y)\right\} .
$$

\section{APPENDIX B}

PROOF OF THEOREM 1

Proof: Having the channel $\mathbf{H}$ given by (2), we denote

$$
\mathbf{W}=\frac{1}{K}\left\{\begin{array}{lll}
\mathbf{H}_{2}^{\mathrm{H}} \mathbf{H}_{1}^{\mathrm{H}} \mathbf{H}_{1} \mathbf{H}_{2} & \text { if } & s=M \\
\mathbf{H}_{1}^{\mathrm{H}} \mathbf{H}_{1} \mathbf{H}_{2} \mathbf{H}_{2}^{\mathrm{H}} & \text { if } & s=K \\
\mathbf{H}_{1} \mathbf{H}_{2} \mathbf{H}_{2}^{\mathrm{H}} \mathbf{H}_{1}^{\mathrm{H}} & \text { if } & s=N .
\end{array}\right.
$$

Next, we write (8) in terms of the eigenvalues of $\mathbf{W}$ by employing Corollary 2 in [23] providing the PDF of an unordered eigenvalue $p(\lambda)$ of the matrix $\mathbf{H}_{2}^{\mathrm{H}} \mathbf{H}_{1}^{\mathrm{H}} \mathbf{H}_{1} \mathbf{H}_{2}$. Specifically, $p(\lambda)$ is given by

$$
p(\lambda)=2 \mathcal{K} \sum_{i=1}^{s} \sum_{j=1}^{s} \frac{\lambda^{\frac{p+2 j+t+i-2 s-3}{2}} K_{t-p+i-1}(2 \sqrt{\lambda}) G_{i, j}}{s \Gamma(p-s+j)},
$$

where $\mathcal{K}$ is given by (12), and $K_{v}(x)$ is the modified Bessel function of the second kind [19, eq. 8.432.1]. Writing (8) in terms of its eigenvalues, we have

$$
\begin{aligned}
& \mathrm{C}^{\mathrm{opt}}\left(\rho, M, N, K, \delta_{\mathrm{t}}, \delta_{\mathrm{r}}\right)=s \int_{0}^{\infty} \log _{2}\left(1+\frac{\frac{\rho}{K M} \lambda}{\frac{\rho \delta_{\mathrm{t}}^{2} \lambda}{K M}+\rho \delta_{\mathrm{r}}^{2}+1}\right) p(\lambda) \mathrm{d} \lambda \\
& =s \int_{0}^{\infty} \log _{2}\left(\left(1+\delta_{\mathrm{t}}^{2}\right) \frac{\rho \lambda}{K M}+\rho \delta_{\mathrm{r}}^{2}+1\right) p(\lambda) \mathrm{d} \lambda \\
& -s \int_{0}^{\infty} \log _{2}\left(\frac{\rho}{K M} \delta_{\mathrm{t}}^{2} \lambda+\rho \delta_{\mathrm{r}}^{2}+1\right) p(\lambda) \mathrm{d} \lambda
\end{aligned}
$$

Substituting (28) into (29) and making use of [19, eq. 7.821.3] after expressing the logarithm in terms of a Meijer G-function according to $\ln (1+x)=G_{2,2}^{1,2}\left(a x \mid \begin{array}{l}1,1 \\ 0,0\end{array}\right)$ [24, eq. 8.4.6.5], the proof is concluded. 


\section{APPENDIX C \\ PROOF OF THEOREM 2}

The focal point is to obtain indirectly the a.e.p.d.f. of $\mathbf{K} / K$ by means of Lemma 3 that includes its Stieltjes transform $\mathcal{S}_{\mathbf{K} / K}$. In other words, we focus on the derivation of the Stieltjes transform of $\mathbf{K} / K$. Specifically, $\mathcal{S}_{\mathbf{K} / K}$ can be obtained by means of its $\eta$-transform after using Lemma 2 . In fact, this lemma allows us to write

$$
x \eta_{\mathbf{K} / K}^{-1}\left(-x \mathcal{S}_{\mathbf{K} / K}(x)\right)+1=0 .
$$

The following proposition provides $\eta_{\mathbf{K} / K}^{-1}(x)$.

Proposition 2: The inverse $\eta$-transform of $\mathbf{K} / K$ is given by

$$
\eta_{\mathbf{K} / K}^{-1}(x)=-\frac{x-1}{x(\beta+x-1)(\gamma(x-1)+1)} .
$$

Proof: Application of the S-transform to (18) and use of the principle of free convolution leads to $\eta_{\mathbf{K} / K}^{-1}(x)$ as

$$
\begin{aligned}
\Sigma_{\mathbf{K} / K}(x) & =\Sigma_{\tilde{\mathbf{N}}_{\mathbf{2}} / K}(x) \Sigma_{\tilde{\mathbf{M}} / K}(x) \Longleftrightarrow \\
\left(-\frac{x+1}{x}\right) \eta_{\mathbf{K} / K}^{-1}(x+1) & =\frac{1}{(\beta+x)(\gamma x+1)},
\end{aligned}
$$

where in (32), we have used Definition 2 and Lemma 1. Herein, we note that $\Sigma_{\tilde{\mathbf{N}}_{\mathbf{2}} / K}(x)$ and $\Sigma_{\tilde{\mathbf{N}}_{1} / K}(x)$ are given by (23) and (24) as

$$
\Sigma_{\tilde{\mathbf{N}}_{\mathbf{2}} / K}(x)=\frac{1}{\gamma x+1}
$$

and

$$
\Sigma_{\tilde{\mathbf{N}}_{1 / K}}(x)=\frac{1}{\beta+x} .
$$

Moreover, it is worthwhile to mention that in (32), we have taken into account the asymptotic freeness between the deterministic matrix with bounded eigenvalues $\tilde{\mathbf{N}}_{2}$ and the unitarily invariant matrix $\tilde{\mathbf{N}}_{1}$. By setting $y=x+1$, i.e., making a change of variables, we lead to (31).

After using (30) and Proposition 2, we lead to the following qubic polynomial after tedious algebraic manipulations

$$
\begin{array}{r}
x^{2} \gamma \mathcal{S}_{\mathbf{K} / K}^{3}-(\beta \gamma-2 \gamma+1) x \mathcal{S}_{\mathbf{K} / K}^{2} \\
-(\beta \gamma-\beta-\gamma+x+1) \mathcal{S}_{\mathbf{K} / K}-1=0 .
\end{array}
$$

This polynomial provides $\mathcal{S}_{\mathbf{K} / K}$, and thus, $f_{\frac{\mathrm{K}}{K}}^{\infty}(x)$ by means of Lemma 3, which is the desired result.

\section{ACKNOWLEDGEMENT}

This work was partially supported by FNR, Luxembourg under the CORE projects "SeMIGod" and "SATSENT".

\section{REFERENCES}

[1] E. Telatar, "Capacity of multi-antenna Gaussian channels," Europ. Trans. on Telecom., vol. 10, no. 6, pp. 585-595, 1999.

[2] D. Gesbert, H. Bölcskei, D. A. Gore, and A. J. Paulraj, "Outdoor MIMO wireless channels: Models and performance prediction," IEEE Trans. on Commun., vol. 50, no. 12, pp. 1926-1934, 2002.

[3] D. Chizhik, G. J. Foschini, M. J. Gans, R. Valenzuela et al., "Keyholes, correlations, and capacities of multi-element transmit and receive antennas," IEEE Trans. on Wireless Commun., vol. 1, no. 2, pp. 361-368, 2002.
[4] J. P. Kermoal, L. Schumacher, K. I. Pedersen, P. E. Mogensen, and F. Frederiksen, "A stochastic MIMO radio channel model with experimental validation," IEEE Journal on Sel. Areas in Commun., vol. 20, no. 6, pp. 1211-1226, 2002.

[5] H. Shin and J. H. Lee, "Capacity of multiple-antenna fading channels: Spatial fading correlation, double scattering, and keyhole," IEEE Trans. on Inform. Theory, vol. 49, no. 10, pp. 2636-2647, 2003.

[6] H. Shin and M. Z. Win, "MIMO diversity in the presence of double scattering," IEEE Trans. Inform. Theory, vol. 54, no. 7, pp. 2976-2996, 2008

[7] C. Zhong, T. Ratnarajah, Z. Zhang, K. K. Wong, and M. Sellathurai, "Performance of Rayleigh-product MIMO channels with linear receivers," IEEE Trans. Wireless Commun., vol. 13, no. 4, pp. 22702281, 2014.

[8] T. Schenk, $R F$ imperfections in high-rate wireless systems: impact and digital compensation. Springer Science \& Business Media, 2008

[9] C. Studer, M. Wenk, and A. Burg, "MIMO transmission with residual transmit-RF impairments," in ITG/IEEE Work. Smart Ant. (WSA). IEEE, 2010, pp. 189-196.

[10] E. Björnson, P. Zetterberg, M. Bengtsson, and B. Ottersten, "Capacity limits and multiplexing gains of MIMO channels with transceiver impairments," IEEE Commun. Lett., vol. 17, no. 1, pp. 91-94, 2013.

[11] A. Papazafeiropoulos, S. K. Sharma, and S. Chatzinotas, "Impact of transceiver impairments on the capacity of dual-hop relay massive MIMO systems," in IEEE Global Communications Conference (GLOBECOM 2015) - Workshop on Massive MIMO: From theory to practice, 2015.

[12] —, "MMSE filtering performance of DH-AF massive MIMO relay systems with residual transceiver impairments," in IEEE International Conference on Communications (ICC 2016) - Workshop on Advanced PHY and MAC Technology for Super Dense Wireless Networks, 2016.

[13] T. Marzetta, "Noncooperative cellular wireless with unlimited numbers of base station antennas," IEEE Trans. Wireless Commun., vol. 9, no. 11, pp. 3590-3600, November 2010.

[14] A. Osseiran, F. Boccardi, V. Braun, K. Kusume, P. Marsch, M. Maternia, O. Queseth, M. Schellmann, H. Schotten, H. Taoka, H. Tullberg, M. Uusitalo, B. Timus, and M. Fallgren, "Scenarios for 5G mobile and wireless communications: The vision of the METIS project," IEEE Commun. Mag., vol. 52, no. 5, pp. 26-35, May 2014.

[15] X. Zhang, M. Matthaiou, E. Björnson, M. Coldrey, and M. Debbah, "On the MIMO capacity with residual transceiver hardware impairments," in Proc. IEEE Int. Conf. Commun. IEEE, 2014, pp. 5299-5305.

[16] E. Björnson, J. Hoydis, M. Kountouris, and M. Debbah, "Massive MIMO systems with non-ideal hardware: Energy efficiency, estimation, and capacity limits," IEEE Trans. Inform. Theory, vol. 60, no. 11, pp. 7112-7139, Nov 2014.

[17] E. Björnson, M. Matthaiou, and M. Debbah, "Massive MIMO with nonideal arbitrary arrays: Hardware scaling laws and circuit-aware design," IEEE Trans. Wireless Commun., vol. 14, no. no.8, pp. 4353-4368, Aug. 2015 .

[18] H. Holma and A. Toskala, LTE for UMTS: Evolution to LTE-Advanced, Wiley, Ed., 2011.

[19] I. S. Gradshteyn and I. M. Ryzhik, "Table of integrals, series, and products," Alan Jeffrey and Daniel Zwillinger (eds.), Seventh edition (Feb 2007), vol. 885, 2007.

[20] S. Chatzinotas and B. Ottersten, "Capacity analysis of dual-hop amplifyand-forward MIMO multiple-access channels," in International Conference on Wireless Communications \& Signal Processing (WCSP), Hunagshan, China, Oct 2012.

[21] A. M. Tulino and S. Verdú, Random matrix theory and wireless communications. Now Publishers Inc., 2004, vol. 1, no. 1.

[22] J. W. Silverstein and Z. Bai, "On the empirical distribution of eigenvalues of a class of large dimensional random matrices," Journal of Multivariate analysis, vol. 54, no. 2, pp. 175-192, 1995.

[23] S. Jin, M. R. McKay, C. Zhong, and K. K. Wong, "Ergodic capacity analysis of amplify-and-forward MIMO dual-hop systems," IEEE Trans. on Inform. Theory, vol. 56, no. 5, pp. 2204-2224, 2010.

[24] A. Prudnikov, Y. Brychkov, and O. Marichev, Integrals and Series: More special functions, ser. Integrals and Series. Gordon and Breach Science Publishers, 1990. [Online]. Available: https://books.google.gr/books?id=OdS6QgAACAAJ 